\title{
Omental transplantation improves surgical outcome of large squamous cell carcinoma: A case report
}

\author{
XIANAN LI ${ }^{1 *}$, ZHENGXIAO OUYANG $^{1,2 *}$, SHUO YANG ${ }^{1}$, ZANJING ZHAI $^{2}$, \\ HAOWEI LI ${ }^{2}$, ZHONG-CHENG $\mathrm{KANG}^{3}$ and $\mathrm{ZAN} \mathrm{LI}^{4}$ \\ ${ }^{1}$ Department of Orthopedics, Hunan Cancer Hospital and The Affiliated Cancer Hospital of Xiangya School of Medicine, \\ Central South University, Changsha, Hunan 410012; ${ }^{2}$ Department of Orthopedics, Shanghai Key Laboratory of \\ Orthopedic Implant, Shanghai Ninth People's Hospital, Shanghai Jiaotong University School of Medicine, \\ Shanghai 200001; ${ }^{3}$ Department of Hepatobiliary Surgery; ${ }^{4}$ Department of Head and Neck Surgery, Hunan \\ Cancer Hospital and The Affiliated Cancer Hospital of Xiangya School of Medicine, \\ Central South University, Changsha, Hunan 410012, P.R. China
}

Received June 10, 2014; Accepted February 26, 2015

DOI: $10.3892 / \mathrm{ol} .2015 .3089$

\begin{abstract}
Cutaneous squamous cell carcinoma (SCC) is an major health issue due to the significant health costs and marked disfigurement following surgical excision. The conventional reconstructive options may not be suitable for patients with large SCCs of the lower part of the leg, due to the regional tissue damage and ischemic environment. The omental transposition flap is a highly vascularized tissue that is resistant to infection and provides a recipient bed for skin grafts. This is the case report of a male patient with a large SCC in the lower part of his right leg, which was treated with extensive resection. The patient subsequently underwent reconstruction using a free omental flap, followed by transplantation of a secondary skin graft. The patient did not experience recurrence or metastasis during the 2 years of follow-up, indicating that, when dealing with postoperative skin cancer defects in an ischemic environment, omental transplantation may be a viable treatment option.
\end{abstract}

Correspondence to: Dr Zhong-Cheng Kang, Department of Hepatobiliary Surgery, Hunan Cancer Hospital and The Affiliated Cancer Hospital of Xiangya School of Medicine, Central South University, 283 Tongzipo Road, Changsha, Hunan 410012, P.R. China E-mail: kangzhongcheng@sina.com

Professor Zan Li, Department of Head and Neck Surgery, Hunan Cancer Hospital and The Affiliated Cancer Hospital of Xiangya School of Medicine, Central South University, 283 Tongzipo Road, Changsha, Hunan 410012, P.R. China

E-mail: zzanli@163.com

*Contributed equally

Key words: squamous cell carcinoma, omental transplantation, skin defect reconstruction, non-melanoma skin cancer, limb-salvage surgery

\section{Introduction}

Squamous cell carcinoma (SCC) of the skin, which develops from the keratinocytes of the epidermis, is the second most common skin malignancy and accounts for $20 \%$ of all skin cancers (1). The incidence of SCC continues to increase (2) and represents a major public health concern due to the associated significant health costs and marked disfigurement following surgical excision. Patients with primary SCC have a good prognosis, however, the long-term prognosis is relatively poor for those with metastatic disease, which has a 10 -year survival rate of $<20 \%$ (2). Clinically, the diagnosis of SCC is confirmed by skin biopsy. At present, treatment modalities include radiotherapy, brachytherapy, electrodesiccaation, cryotherapy and photodynamic therapy, however, surgical excision and Mohs micrographic surgery (MMS) are considered the gold standard treatments for high-risk SCC (3). Despite this, due to the high cost and complicated surgical process, at present MMS is not widely used. The omental flap, a classical reconstructive flap widely used for the treatment of ulcers and defects of the breast, may provide a vascularized recipient bed for skin grafts and thus, may adapt well to an ischemic environment, such as the lower limb (4). In this study, the case of a male patient with a large SCC in the lower part of the right leg, who underwent extensive resection with reconstruction using an omental transposition flap, is presented.

\section{Case report}

A 49-year-old male presented to Hunan Cancer Hospital and The Affiliated Cancer Hospital of Xiangya School of Medicine (Changsha, China) with a ulcerated skin lesion in the lower part of the right leg, for which amputation was suggested by doctors at other hospitals. The physical examination on admission revealed the presence of a $\sim 25 \times 20-\mathrm{cm}^{2}$ cutaneous cauliflower-shaped ulcerated lesion, with a specific odor and exudate, occupying one-third of the lower part of the leg (Fig. 1), with lymphadenopathy of the ipsilateral inguinal 


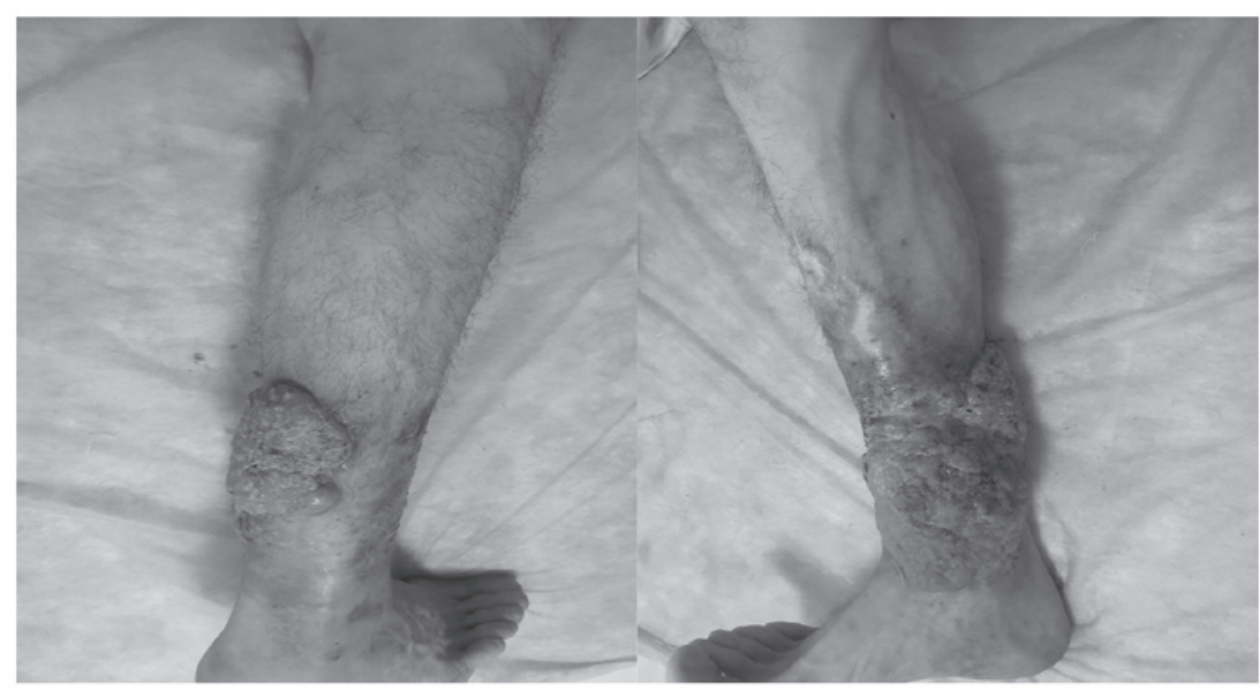

Figure 1. Large ulcerated lesion on the skin of the lower part of the right leg.

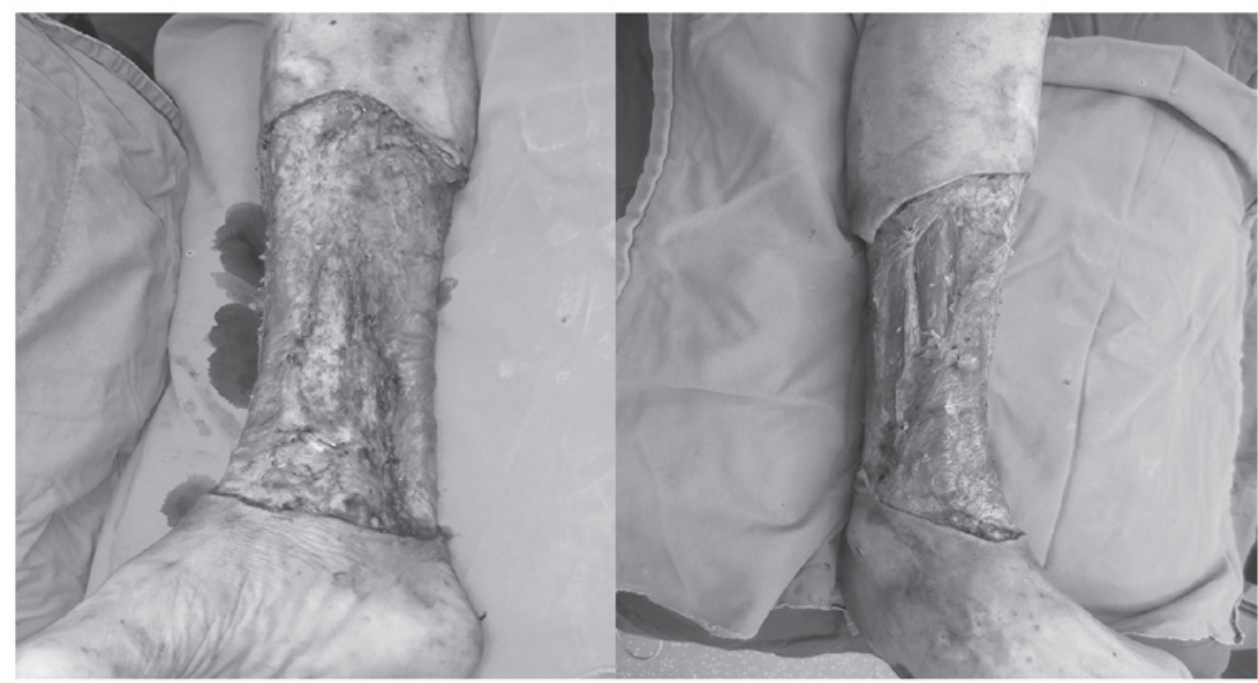

Figure 2. Defect following extensive resection of the skin of the lower part of the right leg.

region. The preoperative pathological examination established the diagnosis of highly differentiated SCC and the bacteriological examination revealed infection by levofloxacin-sensitive Pseudomonas aeruginosa.

Prior to surgery, potassium permanganate was used to soak the limb and the dressing was changed daily. The infection was effectively controlled following administration of antibiotics with high sensitivity. Thereafter, the patient underwent extensive resection of the original lesion (Fig. 2), with additional biopsy of the ipsilateral inguinal lymph nodes, which did not identify lymph node metastasis.

The autologous reconstructive options for the present case were preoperatively discussed in a multidisciplinary team setting involving general and plastic surgeons. It was decided to repair the defect using a free omental flap with a vascular pedicle, as this satisfied the reconstructive requirements, namely covering the defect with healthy, well-vascularized tissue and a reliable flap, while avoiding the long operative time and high cost that are associated with Mohs micrographic surgery (MMS) (5).
The patient was placed in the supine position. Following an upper midline incision, the omentum was carefully mobilized and excised along with the right gastroepiploic pedicle, subsequent to which it was transposed to the debrided leg defect (Fig. 3). The dorsal artery was used as a recipient vessel for the right gastroepiploic artery and anastomosis was performed in an end-to-end manner. The secured omentum was covered with a secondary skin graft that was harvested from the thigh of the patient 10 days after the first operation.

Postoperatively, the patient was prescribed a 2-day course of cefoperazone/tazobactam. Due to persistent wound discharge, the antibiotic therapy was continued for 5 days; by day 5 , the wound discharge had significantly increased. The drainage from the incision was collected for bacteriological examination and the patient was administered antibiotics (ceftazidime and levofloxacin) with high sensitivity and specificity following secondary skin grafting. The skin graft exhibited favorable survival (Fig. 4) and the patient was discharged on the 15th postoperative day. No complications were reported. At the 24-month follow-up examination, there were no signs of 


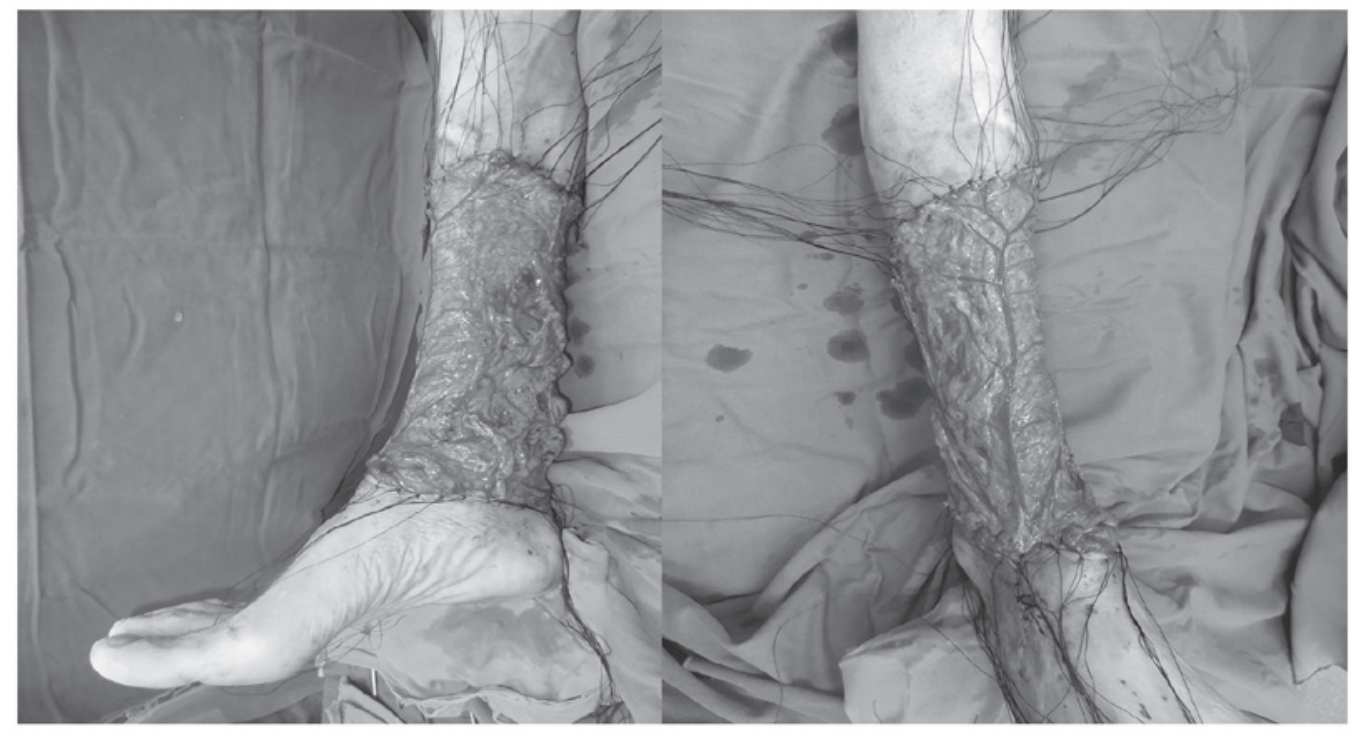

Figure 3. The defect was repaired using a free omental flap.

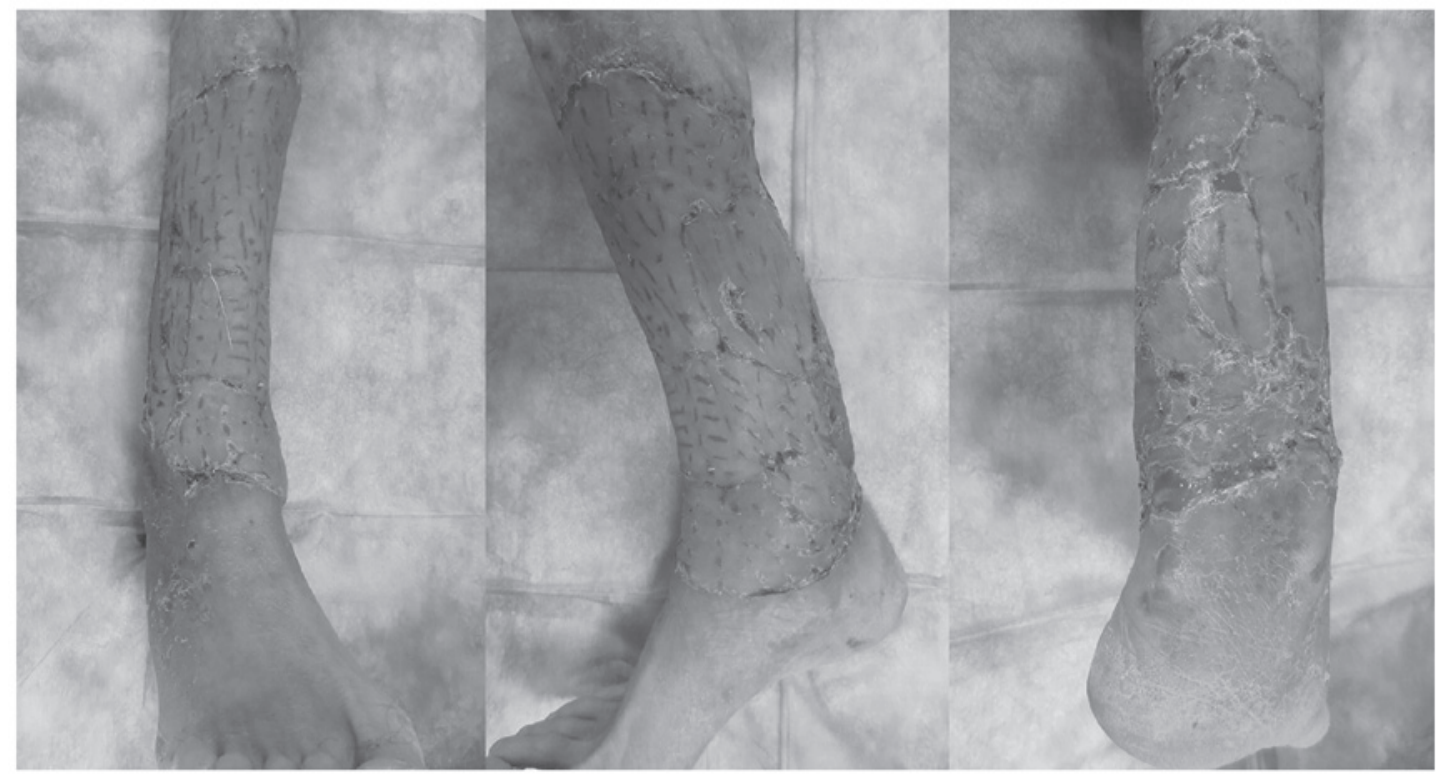

Figure 4. Lower part of the right leg at 15 days following secondary skin transplantation.

disease recurrence and no reported pain or ulceration. Written informed consent was obtained from the patient.

\section{Discussion}

The biological behavior of SCC is determined by several variables (6) and its treatment should be selected based on the lesion size, anatomical location, invasion depth, degree of cellular differentiation and history of previous treatment (7). However, surgery with a predefined excision margin is considered the optimal treatment option for the majority of cutaneous SCC cases. In addition, MMS is recommended for SCCs that are associated with a higher risk or located at anatomical sites requiring maximal tissue conservation for the preservation of function and cosmesis (8). However, MMS is associated with a high cost and long operative duration and no randomized controlled trials have yet been performed to directly compare the two surgical procedures (3).

In the present case, considering the large area occupied by the tumor and the fact that the lesion was located at a region where the likelihood of postoperative skin necrosis and infection is high, the patient was recommended to undergo amputation at other hospitals prior to presenting to our institution. However, amputation markedly affects the quality of life of the patients; therefore, the patient and his family declined this surgical approach.

The omental flap was first described by Kiricta in 1963 (9) and is a classical reconstructive alternative for ulcers of the axilla and breast (10). The omental flap is a highly vascularized tissue that is resistant to infection and adapts well to an ischemic environment (11). Thus, the use of the greater omental flap is considered as a back-up option in complicated cases. At 
present, the free omental flap is commonly used for improving subcutaneous contour and wound coverage, as it provides a vascularized recipient bed for skin grafts.

Considering the absence of lymph node metastasis and the wish of the patient to avoid amputation, the tumor was resected and the defect reconstructed using the free omental transposition flap with secondary skin grafting to achieve satisfactory defect coverage with an adequate volume of well-vascularized tissue. The immunological and angiogenic properties of omental flaps are particularly useful for promoting the healing of defects. As opposed to free fat grafts, which quickly lose volume following transplantation, the vascularized omental flap maintains its volume.

In the present case, the patient did not experience any complications other than early recipient site infection, which was successfully treated with antibiotics. The treatment of SCC aims to completely remove or destroy the tumor and to minimize functional and cosmetic impairment. Thus, limb-salvage surgery with omental transplantation is a potential alternative for such patients in developing countries, where the patients may require a more cost-effective precedure and physicians may lack the special training required for MMS. Furthermore, Chren et al (12) reported that regional recurrence was similar between the two treatment modalities.

The application of omental transplantation in the present case achieved a satisfactory limb contour and also yielded cosmetic and functional outcomes that completely met the requirements of the patient. No local recurrence or metastasis has been reported in the present case; however, further prospective studies are required to determine the specific indications for this technique. In conclusion, omental transplantation may be a viable option for limb-salvage surgery and the reconstruction of defects following resection of large SCCs in regions susceptible to ischemia.

\section{References}

1. McGuire JF, Ge NN and Dyson S: Nonmelanoma skin cancer of the head and neck I: histopathology and clinical behavior. Am J Otolaryngol 30: 121-133, 2009.

2. Alam M and Ratner D: Cutaneous squamous-cell carcinoma. N Engl J Med 344: 975-983, 2001.

3. Lansbury L, Bath-Hextall F, Perkins W, Stanton W and Leonardi-Bee J: Interventions for non-metastatic squamous cell carcinoma of the skin: systematic review and pooled analysis of observational studies. BMJ 347: f6153, 2013.

4. Hartmann CE, Ko LW, Ion L and Jemec B: A difficult case: omental transposition flap reconstruction of a large radionecrotic axillary ulcer in a patient with extensive previous abdominal surgery. J Plast Reconstr Aesthet Surg 66: e66-e68, 2013.

5. Belkin D and Carucci JA: Mohs surgery for squamous cell carcinoma. Dermatologic clinics 29: 161-174, 2011.

6. Barksdale SK, O'Connor N and Barnhill R: Prognostic factors for cutaneous squamous cell and basal cell carcinoma. Determinants of risk of recurrence, metastasis and development of subsequent skin cancers. Surg Oncol Clin N Am 6: 625-638, 1997.

7. Goldman GD: Squamous cell cancer: a practical approach. Semin Cutan Med Surg 17: 80-95, 1998.

8. Stasko T, Brown MD, Carucci JA, et al; International Transplant-Skin Cancer Collaborative; European Skin Care in Organ Transplant Patients Network: Guidelines for the management of squamous cell carcinoma in organ transplant recipients. Dermatol Surg 30: 642-650, 2004

9. Kiricuta I: The use of the great omentum in the surgery of breast cancer. Presse Med 71: 15-17, 1963 (In French).

10. Katz MH: Nonmelanoma skin cancer. Md Med J 46: 239-242, 1997.

11. Panje WR, Pitcock JK and Vargish T: Free omental flap reconstruction of complicated head and neck wounds. Otolaryngol Head Neck Surg 100: 588-593, 1989.

12. Chren MM, Linos E, Torres JS, Stuart SE, Parvataneni R and Boscardin WJ: Tumor recurrence 5 years after treatment of cutaneous basal cell carcinoma and squamous cell carcinoma. J Invest Dermatol 133: 1188-1196, 2013. 